\title{
Transatlantica
}

Revue d'études américaines. American Studies Journal

\section{Blakelock : sortie de nuit}

« The Unknown Blakelock» à la National Academy of Design, New York, 25 septembre 2008 -5 janvier 2009.

\section{Hélène Valance}

\section{Q OpenEdition}

1 Journals

\section{Édition électronique}

URL : https://journals.openedition.org/transatlantica/4317

DOI : $10.4000 /$ transatlantica. 4317

ISSN : 1765-2766

Éditeur

Association française d'Etudes Américaines (AFEA)

Référence électronique

Hélène Valance, «Blakelock : sortie de nuit », Transatlantica [En ligne], 1 | 2009, mis en ligne le 02 septembre 2009, consulté le 16 septembre 2021. URL : http://journals.openedition.org/transatlantica/ 4317 ; DOI : https://doi.org/10.4000/transatlantica.4317

Ce document a été généré automatiquement le 16 septembre 2021.

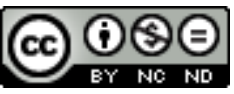

Transatlantica - Revue d'études américaines est mise à disposition selon les termes de la licence Creative Commons Attribution - Pas d'Utilisation Commerciale - Pas de Modification 4.0 International. 


\title{
Blakelock : sortie de nuit
}

\author{
«The Unknown Blakelock» à la National Academy of Design, New York, \\ 25 septembre 2008 -5 janvier 2009.
}

\section{Hélène Valance}

\section{«An American Van Gogh » ${ }^{1}$}

1 Tandis que le Museum of Modern Art attire les foules avec son Van Gogh and the Colors of the Night, la National Academy of Design se penche sur un artiste du nocturne américain, Ralph Albert Blakelock (1847-1919). Fondation mère de l'art américain au XIX ${ }^{\text {ème }}$ siècle, l'institution, logée dans un petit immeuble voisin du Guggenheim, n'a pas aujourd'hui l'ampleur ni la popularité du MoMA, surtout depuis la récente controverse autour de la vente d'une partie de sa collection permanente ${ }^{2}$. Et Blakelock, si l'on a pu lui attribuer comme à Van Gogh l'aura du génie maudit, ne partage pas le succès de ce dernier. Tout ce que l'on connaît de Ralph Albert Blakelock, bien souvent, ce sont ses scènes de l'Ouest et ses moonlights, et quelques détails d'une biographie tourmentée. Plus ou moins autodidacte, Blakelock développe une réelle maturité artistique au terme de deux longs voyages dans l'Ouest américain et les Caraïbes. De retour à New York, il attire l'attention de nombreux connaisseurs, mais ne parvient jamais à une notoriété suffisante pour entretenir une famille toujours plus nombreuse. Dès 1890, il montre des signes de troubles mentaux. En 1899, il est interné pour "dementia praecox», et passera le reste de sa vie en asile psychiatrique. Ses œuvres, en particulier ses paysages nocturnes, se vendent pourtant de mieux en mieux, atteignant des prix record. Mais, pour ajouter à ce tableau pathétique, Blakelock se voit dérober les recettes de ces ventes, tandis qu'usurpateurs et imposteurs se multiplient, inondant le marché de "Fakelocks». Obscure et lunatique, la figure romancée de Blakelock domine la majorité des études qui lui sont consacrées. S'il apparaît au cœur du Moon Palace de Paul Auster, c'est encore en tant que génie obscur et voué à le rester. L'exposition de la National Academy of Design veut au contraire sortir Blakelock de l'ombre, et donner à voir un échantillon plus complet de son œuvre. The Unknown Blakelock, initialement présentée à la Sheldon Memorial Art Gallery de l'Université de Nebraska, comprend peu d'œuvres issues des musées de la côte est: en soi une 
découverte pour le public newyorkais. L'exposition couronne les travaux de Norman Geske, professeur émérite à l'Université de Nebraska, auteur de Beyond Madness : The Art of Ralph Blakelock(2007). Geske, qui a consacré plus de 25 ans à la constitution du Nebraska Blakelock Inventory, tente d'ouvrir de nouvelles perspectives sur l'artiste et son œuvre, insistant en particulier sur la variété de ses sujets et ses innovations techniques. Confiné au rôle stéréotypé de l'artiste marginal, Blakelock apparaît en effet comme un artiste doublement méconnu.

\section{Histoire(s) d'un « outsider »}

2 Les raisons de cette méconnaissance sont multiples. Blakelock est souvent présenté comme un «moonlighter» qui peindrait au noir, en marge de la légitimité professionnelle. Venu à l'art par des voies détournées (quelques cours de dessin à la Free Academy, ancêtre du New York City College), Blakelock n'a visiblement eu ni maître ni disciple attitré. Il ne reste cependant pas étranger aux cercles artistiques de son temps : de 1867 à 1873, il expose régulièrement à la National Academy et dans les années 1880 il occupe un studio dans le célèbre Tenth Street Studio Building où travaillent également plusieurs de ses amis. Les débuts de sa carrière sont influencés par le style de la Hudson River School, et il semble difficile de concevoir que les œuvres de l'école de Barbizon, alors très en vogue dans les galeries newyorkaises, soient passées inaperçues de Blakelock. At Nature's Mirror, comme un écho distant du Concert Champêtre du Titien,confirme que Blakelock connaissait les maîtres. L'image d'un Blakelock « autodidacte » perdure pourtant, car elle permet d'expliquer aisément son style peu conventionnel. L'artiste ainsi isolé peut aussi plus facilement être présenté sous les traits de l'individu de génie, incompris de ses contemporains, une vision qui coïncide avec la schizophrénie dont souffre Blakelock à partir des années 1890. Les nocturnes de la fin de sa vie, en particulier, encouragent la critique et le public à voir en Blakelock un peintre de l'« intériorité » et de la "mélancolie », ressassant tous les poncifs du "poète » lunatique sans réellement considérer son art. Le titre de Geske, Beyond Madness, indique à quel point cette interprétation a dominé jusque récemment la lecture de Blakelock. Pour reprendre en les détournant les mots du critique Sadakichi Hartmann, Blakelock a en effet été « obscurci par la folie »3. Après-guerre, il refait brièvement surface, génie maudit tout à coup devenu génie de son peuple, dans une exposition que Lloyd Goodrich lui consacre au Whitney Museum. Blakelock est relégué aux marges du New York artistique et cosmopolite pour être érigé, par contraste, en " véritable » artiste américain. Aux côtés de Ryder, Blakelock est alors vu comme l'un des "originaux» fondateurs de la modernité américaine, un artiste qui s'inspire plus de la peinture "primitive» des tribus de l'Ouest que de la tradition européenne. Emancipé des contraintes académiques ou sociales, Blakelock serait ainsi plus fidèle à la nature qu'il dépeint, excentrique porté au rang de prophète de l'identité américaine. Sans oblitérer le caractère profondément individuel de l'œuvre de Blakelock, l'exposition de la National Academy a le mérite de replacer ce dernier dans son temps, et de mettre en sourdine les réflexions sur l'isolation, professionnelle ou mentale, de l'artiste. 


\section{Zones d'ombre}

D'autres obstacles s'opposent cependant au travail de l'historien ou du commissaire d'exposition. L'abondance des faux tout d'abord, qui a très tôt découragé les acheteurs et, à leur suite, les spécialistes de l'art. Le Nebraska Blakelock Inventory se veut une réponse à ce problème, définissant des critères d'analyse des tableaux de l'artiste départageant clairement les tableaux authentiques des imitations. La rareté des documents laissés par l'artiste lui-même, le fait que la plupart des tableaux ne sont pas datés, ni même signés, ont sans doute contribué à l'inflation de "Fakelocks". Blakelock, forcé par la pauvreté, s'est également souvent vu obligé de produire rapidement des peintures de moindre qualité, adultérant lui-même sa propre production. Même lorsque l'authenticité d'un tableau est vérifiée, il reste difficile à exploiter, faute de documentation directe. Les commentateurs se trouvent bien souvent contraints à recourir aux critiques et témoignages de l'époque, avec les distorsions que cela implique. Le secret de Blakelock semble enfoui jusque dans sa peinture: de nombreuses toiles sont en très mauvais état, et ne ressemblent parfois guère à ce qu'elles étaient il y a plus d'un siècle. Blakelock multipliait en effet les expérimentations, râpait ses toiles, les passait à l'eau, empilait les couches de peinture sans leur laisser le temps de sécher, concoctait ses propres vernis et pigments. Il faut aussi savoir reconnaitre les traces du temps dans les surfaces gonflées, cloquées, craquelées qui ont contribué à la réputation du peintre fou. La noirceur de certaines toiles ou leur coloris verdâtre tient plus souvent au vieillissement de ses vernis au bitume qu'à sa palette originale. Sunset, Navarro Ridge, California Coast semble par exemple avoir perdu la splendeur première que laisse attendre le titre du tableau. La mystérieuse silhouette féminine qui se fond dans le paysage de Going to the Spring (1870-79, Valley National Bank of Arizona, Fine Arts Department) était sans doute plus nette sous le pinceau de Blakelock, comme le suggère le pointillé blanc de son collier. Blakelock reste dans une large mesure un inconnu, enfoui sous cette peinture altérée. Quoi de plus déroutant pour l'admirateur contemporain de l' «obscur» Blakelock de lire qu'il se disait « le meilleur coloriste depuis Titien »?

\section{Eclipser le lunatique}

4 A les regarder de plus près, pourtant, les nocturnes de Blakelock se révèlent plus radieux qu'obscurs. En contraste avec les bleus sombres de Winslow Homer ou les tonalités froides et voilées de Whistler, la peinture de Blakelock déploie les nuances du clair de lune. Moonlight Sonata ${ }^{5}$ (1889-92, Museum of Fine Arts, Boston) offre un bel exemple de la technique de Blakelock: de l'astre lunaire fiché au centre du tableau aux masses sombres des feuillages au premier plan, le tableau distille d'infinies variétés de lumière. L'atmosphère vaporeuse de l'étang se raffine à mesure que l'œil se dirige vers le centre de la composition, où la lune perce un trou brillant. A travers les couches successives de peinture et de vernis coloré filtre une lueur polie qui infuse la surface du tableau, travaillée à l'extrême. Moonlight Sonata fonctionne presque comme une illusion d'optique, piégeant le regard dans sa source lumineuse. Le spectateur se trouve attiré au cœur du tableau comme les phalènes de Japanese Lantern with Moths (n.d., collection de M. et Mme. Walter Blakelock Wilson, Arizona). L'œil ébloui revient au lacis de la végétation qui structure le paysage, pour découvrir un trait singulier de l'art de 
Blakelock: le peintre applique en premier sur le canevas les masses noires des frondaisons, pour ensuite y découper les taches de lumière. Un travail de dentelle en couleur, comme le suggère le titre d'un autre nocturne Moonlight, Silver and old Lace (1880-1890, Santa Barbara Museum of Art), et surtout un art en positif, qui progresse de l'obscurité à la lumière. On est tenté de rapprocher cette peinture radieuse des écrits de Swedenborg, dont Blakelock était un disciple, sur la lumière et la phosphorescence. De l'atmosphère placide de Moonlight Sonata on passe, dans la salle suivante, au ciel hachuré, quasi hiéroglyphique de Moonlight (années 1880, Sheldon Memorial Art Gallery). C'est ce dernier que les organisateurs de l'exposition ont choisi pour couverture de leur catalogue, une façon d'attirer l'attention sur les explorations et innovations techniques de Blakelock sans toutefois trop s'éloigner de ses fameux paysages de clair de lune. St. Gabriel's Grotto, Isle of Jamaica (1872, Parthenon Museum, Nashville), un paysage hors du commun pour la peinture américaine de l'époque, laisse entrevoir le maitre coloriste que fut Blakelock. Les verts luxuriants et les tons bruns de la roche s'entremêlent dans un style très fluide, quasi impressionniste, qui marquent son affranchissement vis-à-vis des conventions de l'Hudson River School. Les figures humaines, minuscules, et les détails du feuillage disparaissent sous les coups de pinceaux, fondus dans la matière du tableau. Que l'on compare le Niagara Falls (1857, Corcoran Gallery, Washington) de Frederic Church à Maiden in the Mist (n.d., Grey Collection, Brookville) : la surface lisse de l'eau et les vapeurs de la chute chez Church n'ont pas la violence de la cataracte multicolore de Blakelock, qui se soulève littéralement sur la toile, et semble répondre à l'appel de la mystérieuse figure féminine au premier plan. Restes de son passage chez les Indiens ou influence des théories de Swedenborg, la nature s'anime dans les paysages de Blakelock, et devient tout entière mouvement, abolissant la séparation entre le sujet et le monde qui l'entoure, comme dans le remarquable Pégasus (avant 1913, Denver Art Museum). Blakelock innove par son traitement de la couleur et par la gamme de ses expérimentations techniques, mais aussi par son approche des sujets habituels de la peinture américaine. Loin des formats spectaculaires et des épopées dont l'homme blanc est le héros, les paysages de l'Ouest chez Blakelock se réservent des formats modestes et intimistes, et la présence humaine, souvent lointaine, est presque toujours indienne. Plus surprenant encore, Blakelock ne se contente pas de retracer sur la toile ses souvenirs de voyage, mais choisit de dépeindre des sujets inédits. Ce "painter's painter » explore ainsi surfaces et matières dans sa série de Shanties, qui apparaît sans doute comme l'un des premiers paysages de friche urbaine. L'exposition, c'était à prévoir, reprend en partie à son compte la thèse de Goodrich qui veut faire valoir Blakelock comme le prédécesseur de la peinture moderne. Il est vrai que certains paysages, rendus indéchiffrables sous les agglomérats de peinture, semblent tenir autant du Pollock que du Blakelock, et que Franz Kline considérait ce dernier comme son peintre préféré. Sans se hasarder à spéculer sur le rôle joué par Blakelock dans l'avènement de la modernité américaine, on peut toutefois regarder les œuvres présentées à la National Academy comme le témoignage d'un artiste profondément curieux et novateur, qui a su affirmer son indépendance dans une peinture aux facettes multiples. Blakelock, dont on a trop souvent noirci le portrait, apparaît moins comme un peintre marginal que comme un artiste avisé, un explorateur de frontières, de l'Ouest sauvage aux banlieues de Manhattan. 


\section{BIBLIOGRAPHIE}

Daingerfield, Elliott, Ralph Albert Blakelock, New York, 1914.

Davidson, Abraham A., " The Wretched Life and Death of an "American Van Gogh" ", Smithsonian 1987, vol. 18, pp 80-91.

-------, Ralph Albert Blakelock, University Park, PA: Pennsylvania State University Press, 1996.

Geske, Norman A., Beyond Madness: The Art of Ralph Blabelock, 1847-1919. Lincoln, NE: University of Nebraska Press, 2007.

Geske, Norman A., Mitchell, Mark D., Siedell, Daniel A., Driesbach, Janice, The Unknown Blakelock. Lincoln, NE: Sheldon Memorial Art Gallery, 2008.

Glyn, Vincent, The Unknown Night: the Genius and Madness of R.A. Blakelock, An American Painter. New York: Grove Press, 2003.

Hartmann, Sadakichi, A History of American Art. Boston: L.C. Page and Co, 1901.

\section{NOTES}

1. Davidson, "The Wretched Life and Death of an "American Van Gogh" ", Smithsonian 1987, vol. 18, pp 80-91.

2. Voir par exemple l'article de Robin Pogrebin dans le New York Times du 22 décembre dernier : http://www.nytimes.com/2008/12/23/arts/design/23acad.html?

$\mathrm{scp}=2 \& \mathrm{sq}=$ national\%20academy\%20of\%20design\&st=cse

3. Sadakichi Hartmann, A History of American Art, 1901, cité par Mark D. Mitchell dans le catalogue de l'exposition.

4. "The most capable colorist since Titian» Lettre de Lewis W. Francis à Lloyd Goodrich, 26 février 1947, Blakelock Archives, Frick Art Reference Library, New York.

5. http://www.mfa.org/collections/search_art.asp?

coll_keywords=moonlight+sonata\&submit. $x=0$ \&submit. $y=0$

\section{INDEX}

Thèmes : Trans'Arts

\section{AUTEUR}

\section{HÉLÈNE VALANCE}

Université Paris VII, Institut d'Etudes Anglophones Charles V 